\title{
LOS CAMBIOS DEL COMERCIO ÉTNICO EN LOS SUBURBIOS DE BARCELONA (2004-2011)
}

JORDI NOFRE ${ }^{1}$

\begin{abstract}
Resumen - Este artículo pretende comprobar empíricamente y a través de un enfoque teórico-metodológico eminentemente geográfico la posible existencia de un proceso "etnogentrificador" en el sector oriental del Distrito de Collblanc - La Torrassa de L'Hospitalet de Llobregat (Barcelona). Para ello, se partirá de una breve síntesis de los estudios de 2004 - publicados en Nofre (2006) - para después presentar la actualización a fecha de 2011 de esta cartografía del paisaje comercial étnico minorista en esta área suburbana y dilucidar acerca de un posible abuso del concepto etnogentrificación en el contexto urbano mediterráneo.
\end{abstract}

Palabras clave: Comercio minorista, etnogentrificación, renovación urbana, distrito de Collblanc - La Torrassa, L'Hospitalet de Llobregat, Barcelona.

Resumo - MUdANÇAS NO COMÉRCIO RETALHISTA ÉTNICO NOS SUBÚRBIOS DE BARCELONA (2004-2011). DEBATE SOBRE PROCESSOS DE ETNOGENTRIFICAÇÃO. Este artigo explora o processo de etnogentrificação num bairro operário dos subúrbios de Barcelona, já citado nalguns meios de comunicação locais. Depois de se resumirem os resultados de uma investigação feita em 2004 (publicados em 2006), sobre as mudanças urbanas e sociais na área de estudo, comparam-se esses dados com os de 2011. Esta análise permite salientar que há abusos no uso do termo "etnogentrificação" no contexto específico das cidades mediterrâneas.

Palavras-chave: Comércio étnico, etnogentrificação, renovação urbana, distrito de Collblanc - La Torrassa, L'Hospitalet de Llobregat, Barcelona.

\begin{abstract}
CHANGES IN THE ETHNIC RETAIL TRADE IN THE SUBURBS OF BARCELONA (2004-2011). A DEBATE ABOUT PROCESSES OF ETHNOGENTRIFICATION. This paper aims to explore an early process of ethnogentrification, previously reported by the local media, in a working-class suburban district of Barcelona. Following a brief description of the research carried out in 2004 (published in 2006) on the urban and social changes in this study area, this paper presents a comparative analysis between the continuities and changes on 'retails-
\end{abstract}

Recebido: Janeiro 2012. Aceite: Junho 2013.

1 Centro de Estudos de Sociologia da Universidade Nova de Lisboa (CESNOVA), Faculdade de Ciências Sociais e Humanas, Universidade Nova de Lisboa. E-mail: jnofre@fcsh.unl.pt 
cape' in the period of 2004 to 2011, by updating its ethnic-retail cartography. Comparative results will allow to opening up a debate about some lexical abuses on the use of the term 'ethno-gentrification' in the specific context of Mediterranean cities.

Keywords: Ethnic Retail trade, ethnogentrification, urban renewal, Collblanc - La Torrassa County, L'Hospitalet de Llobregat, Barcelona.

Résumé - MODIFICATIONS DU COMMERCE DES MINORITÉS ETHNIQUES DANS LA BANLIEUE DE BARCELONE (2004-11). On étudie le processus d'ethnogentrification dans un quartier ouvrier de la banlieue de Barcelone, dont les media locaux ont parlé. Après la présentation d'une recherche faite en 2004 et publiée en 2006, sur les changements urbains et sociaux de cette aire d'étude, on compare ces données avec celles de 2011. L'analyse montre que l'utilisation du terme ethnogentrification est abusive, dans le contexte urbain spécifique des villes de l'Europe méditerranéenne.

Mots-clés: Commerce ethnique, ethnogentrification, rénovation urbaine, Collblanc - La Torrassa, L'Hospitalet de Llobregat, Barcelona.

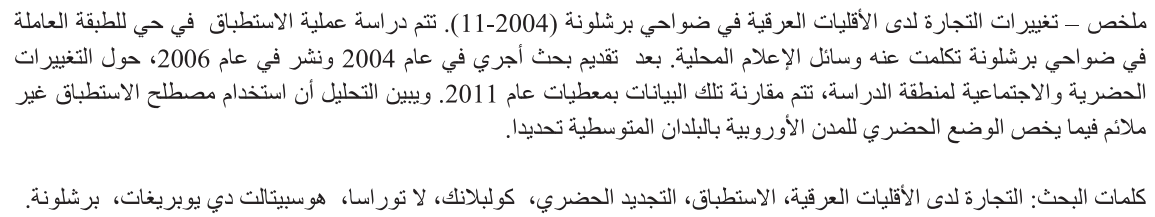

\section{DE LA GEOGRAFÍA DE LA «ETNICIDAD»A LA ETNOGENTRIFICACIÓN}

Martes, 14 de diciembre de 2010. En su sección diaria Vivir Barcelona, el rotativo barcelonés La Vanguardia titulaba: "Adiós al pequeño Guayaquil". Y en subtítulos: "La crisis económica reduce la todavía amplia presencia de ciudadanos ecuatorianos en L'Hospitalet. Los pakistaníes, mucho más competitivos, comienzan a conquistar tejido comercial más allá del Ravali'”. Raúl Montilla y Luís Benvenuty, redactores de la noticia, explicaban como la crisis económica en L'Hospitalet de Llobregat (ciudad suburbial del Área Metropolitana de Barcelona) se está cebando especialmente en el colectivo ecuatoriano, caracterizado por individuos mucho más individualistas que sus conciudadanos pakistaníes e hindúes, los cuales cuentan con amplias redes de autoayuda no solamente a escala local sino supranacional. De hecho, que los diferentes colectivos inmigrantes tiendan a especializar su ocupación laboral según género y rama profesional puede facilitar la generación de redes sociales consolidadas; pero en contextos de crisis económica y financiera relacionada (como en el contexto español) con la burbuja inmobiliaria y la falta de un tejido productivo innovador, ello puede facilitar un "desclasamiento en bloque", el cual, no cabe duda, también afecta a colectivos autóctonos. En el caso del colectivo ecuatoriano de L'Hospitalet de Llobregat, especializado laboralmente en el sector de la 
construcción, este "desclasamiento" se produce a partir de elevados índices de desempleo y endeudamiento bancario ${ }^{\mathrm{iii}}$.

No cabe duda que lo que para unos puede resultar un contexto socioeconómico cotidiano ciertamente difícil, para otros puede convertirse en una fuente de oportunidades. La misma noticia citada en La Vanguardia señalaba cómo - según Huma Jamshed, fundadora de la primera asociación catalana de mujeres pakistaníes - la ciudad de L'Hospitalet “(...) siempre ha gustado a los pakistaníes y, a medida que el Raval se ha ido saturando y la crisis ha apretado las tuercas, desde hace un par de años, L'Hospitalet se ha perfilado como el lugar ideal para volver a empezar. (...) Tiene mucha población, clientes potenciales y los alquileres de los locales comerciales y de los pisos son más económicos que los de Barcelona".

En muchas ciudades anglosajonas, la concentración de comercio minorista regentado por inmigrantes en barrios ampliamente habitados por clases trabajadoras ha constituido uno de los fenómenos más característicos de la segunda mitad del siglo XX. En el caso de las ciudades europeas mediterráneas, el comercio étnico constituye hoy día una de las tónicas dominantes del paisaje comercial minorista tanto de las áreas degradadas de los centros históricos como de aquellos barrios de clase trabajadora caracterizados, generalmente a aunque no en todos los casos, por una densidad poblacional elevada y una edificación compacta. Raulin (2000) nombra esta concentración espacial de comercio regentado por inmigrantes como "centralidad minoritaria", mientras que Ferrer Regalés (2006) lo hace bajo la propuesta nominativa "centralidades interétnicas". Sea como fuera, el hecho es que, tal como sugiere Barth (1969), los grupos étnicos tratan de diferenciarse de otros colectivos a partir, fundamentalmente, de rasgos socio-culturales. Pero sobretodo, lo hacen a partir de prácticas sociales diferenciadas. En el caso de Barcelona - y a modo de ejemplo - el ocio nocturno constituye un mecanismo de distinción/exclusión social de notable importancia (Nofre, 2011). Pero también lo es el establecimiento de circuitos comerciales minoristas diferenciados étnicamente (y socialmente).

A pesar de la multiplicidad y de la gran diversidad de estudios realizados desde los campos de la geografía, la antropología y la sociología urbanas, el estudio del comercio "étnico" en las Ciencias Sociales no se restringe exclusivamente a los últimos quince años. Uno de los primeros autores en estudiar específicamente la formación de tejido comercial minorista regentado por inmigrantes fue Edna Bonacich (1973), quien expuso las bases economicistas de la creación de las redes de solidaridad y capital social de los inmigrantes. Focalizando en el caso de los colectivos caribeños emigrados al área metropolitana de Nueva York en los años sesenta y setenta del siglo XX, las investigaciones sucesivas de Bonacich contribuyeron de manera significativa a difundir y a la vez asentar las perspectivas teóricas economicistas sobre los determinantes y los modos de "incorporación" de los inmigrantes en los respectivos países y ciudades de acogidaiv.

El abordaje del fenómeno inmigratorio desde la óptica de los cambios en la economía local fue uno de los principales marcos teórico-conceptuales que los geógrafos adoptaron en el estudio de los cambios en el paisaje comercial minorista ur- 
bano de las ciudades sobre todo norteamericanas. Así, la "geografía de la etnicidad" apareció como una de las aproximaciones al estudio del comercio étnico que adoptaron carácter incluso subdisciplinar, destacando las aportaciones de Wilson y Portes (1980) sobre la estructura productiva del enclave étnico de Little Habana de Miami, o incluso también la de MaMung y Guillon (1986) sobre el tejido comercial minorista construido por las minorías étnicas del área metropolitana de Paris.

La evolución a lo largo de la década de los años noventa del estudio de la economía de los enclaves étnicos urbanos en las ciudades capitalistas occidentales fue considerado por algunos autores de suma importancia para lograr una explicación fehaciente de la organización social, económica (y política, sin duda alguna) de cualquier grupo étnico localizado más allá de las fronteras de su región de origen. En este sentido, destaca el trabajo de Min Zhou (1992) quien, esta vez desde la sociología urbana, señalaba como la presencia de negocios de emprendedores chinos en la Chinatown de Manhattan evitaba en cierta medida su conversión en un ghetto más de la Gran Nueva York. A pesar de olvidar las estrategias de renovación urbana adoptadas por el gobierno de la ciudad y el gobierno federal del estado de Nueva York a lo largo de la segunda mitad de la década de 1980 (Bryman, 2004; Harvey, 2005), la aportación de Zhou reviste de notable importancia para la evolución más reciente de la relación entre etnicidad y espacio urbano.

En este sentido, tanto Christian Kesteloot y Pascale Mistiaen (1997) como Robert Kaplan (1998) ya advertían como la presencia de negocios de inmigrantes que necesariamente requerían una demanda coétnica local significativa y constante podría indicar el fracaso en la consecución de la integración (o asimilación) social y espacial del grupo étnico en cuestión respecto la sociedad y el país de acogía. Ya a finales de los años noventa, y con uno de los últimos episodios emigratorios de Latinoamérica ya iniciados, sociólogos y geógrafos urbanos como el mismo Kaplan (1998), Robert Kloosterman, Joanne van Der Leun y Jan Rath (1999) e Ivan Light y Steven Gold (2000) destacaron de manera clara y concisa las relación espacial entre barrios con porcentajes significativos de inmigrantes residentes y una elevada concentración de negocios étnicos. Rekers y Ronald van Kempen (2000) también corroboraron esta consideración, señalando como las concentraciones residenciales de inmigrantes y de negocios étnicos muestran con frecuencia una asociación geográfica.

Durante los últimos diez años se han sucedido un número muy notable de estudios de caso particulares acerca de la "geografía de la etnicidad". En un contexto internacional, cabe destacar la investigación sobre la Little Italy de Manchester de Ian Taylor (2000), el barrio de San Lorenzo de Florencia (Loda y Mancini, 2003), la de Jerome Krase y TarryHum (2006) sobre la concentración de comercio minorista chino en el barrio romano del Esquilino; la de Giles A. Barret y David McEvoy (2006) sobre la Curry Mile en Manchester o la de Adelina Miranda (2007), sobre la concentración de comercio minorista chino en la plaza Garibaldi de Nápoles, entre muchísimos otras. Mención especial merece el trabajo de Wei Li, Gary Dymski, Maria W.L. Chee, Hyeon-Hyo Ahn, Carolyn Aldana y Yu Zhou (2006) sobre el tejido 
comercial chino en Los Ángeles. Precisamente la capital del Estado norteamericano de California constituyó el marco geográfico principal del estudio de James M. Smith (2006) sobre el proceso de etnogentrificación ("ethno-gentrification", en original) en el barrio del Little Tokyo de Los Ángeles liderado por el asentamiento de empresas multinacionales japonesas. Precisamente éste es el tipo de "etnogentrificación" que Brian J. Godfrey (2007) identifica como una de las principales estrategias del gobierno local neoyorquino para la revitalización del barrio de Harlem durante los años noventa a través de la venida al barrio de individuos pertenecientes a la clase media-alta negra. En el contexto español, cabe destacar la escasez de producción bibliográfica sobre el comercio étnico de carácter minorista, aunque cabría destacar los estudios sobre la concentración de negocios chinos en el barrio de Fort Pienc en Barcelona (Beltrán, 2000; Beltrán y Sáiz, 2007), y en el Barrio del Raval de Barcelona (Serra, 2006; Valenzuela, 2010), en los municipios almerienses de El Ejido, Roquetas de Mar y Almería (Arjona y Checa, 2005), en el barrio madrileño de Lavapiés (Riesco, 2007), en la ciudad de Valencia (Torres, 2007) e incluso en las Islas Canarias (López Sala y Esteban Sánchez, 2010).

De hecho, la etnogentrificación describe el proceso por el cual en un espacio urbano determinado - normalmente con una morfología urbana caracterizada por una tipología edificatoria compacta tradicional (independientemente de su contexto geográfico) y/o destinada décadas atrás a acoger a las primeras oleadas inmigratorias y localizado o bien en el centro de la ciudad o bien en sus suburbios más próximos - se ve inmerso en una revitalización étnica la cual no tiene por qué implicar necesariamente la expulsión de antiguos residentes de menor capacidad económica con que se suele asociar el concepto "gentrification" (Airriess, 2006). Contrariamente a estas tesis encontraríamos a autores como Steve Shaw et. al (2004), y Sergi Martínez Martínez Rigol (2001), los cuales sostienen, en sus respectivos estudios de caso del barrio londinense de Tower Hamlets y del barcelonés Raval, como la etnogentrificación viene acompañada de una expulsión de antiguas capas tradicionales, autóctonas o no, fenómeno que en el caso de Barcelona es promovido desde la misma administración pública.

\section{OBJETIVOS, OBJETO DE ESTUDIO Y METODOLOGÍA}

La lectura de la noticia citada en las primeras líneas de este artículo sugirió la realización de un trabajo comparativo, de certificación acerca del estado del proceso socioeonómico el cual constituía el principal objeto noticiado. En Diciembre de 2004 realicé un trabajo de campo cualitativo en el que fueron cartografiadas las infraviviendas donde vivían inmigrantes principalmente latinoamericanos en el margen oriental del distrito de Collblanc - La Torrassa de L'Hospitalet de Llobregat, este último, municipio suburbano adyacente a la capital catalana, Barcelona. Además, fueron cartografiados aquellos elementos del paisaje urbano que podían indicar la existencia de un proceso de etnificación del comercio urbano minorista y la aparición 
de elementos "proto-gentrificadores" (Nofre, 2006). Los resultados fueron publicados en el libro coordinado por Carles Carreras y Ana Fani titulado "Barcelona y São Paulo, cara a cara. Procesos metropolitanos a la hora de la globalización".

Lo que este artículo pretende es certificar la existencia o no de un proceso de etnogentrificación en esta área suburbana de L'Hospitalet de Llobregat, ciudad localizada en la mitad sur de la primera corona metropolitana de Barcelona (Catalunya), a través de los cambios producidos en el paisaje comercial minorista en el sector oriental del Distrito de Collblanc - La Torrassav en los últimos seis años desde aquel trabajo de campo realizado a finales de 2004. Para ello se tomará como punto de partida los resultados obtenidos entonces en lo que se refiere a la etnificación del paisaje comercial minorista de esta área considerada. Una vez presentado el objeto de estudio y los resultados obtenidos en ese primer estudio se procederá a presentar los resultados de un nuevo trabajo de campo siguiendo la misma metodología que se utilizó en su día, con el objetivo de homogeneizar al máximo la comparación temporal de los datos cuantitativos obtenidos mediante trabajo de campo observacional. Su tratamiento geoestadístico fue complementado con la realización de 19 entrevistas informales off the record ${ }^{\mathrm{vi}}$ 7 propietarios de comercio minorista étnico de nacionalidad pakistaní, 6 hindúes (todos ellos de la región de Punjab), 5 entrevistas a residentes autóctonos consumidores habituales de comercio minorista y 1 director de oficina bancaria, la principal radicada en el barrio de La Torrassa (por motivos expresos del entrevistado no se especifica la entidad bancaria en este texto). Todas estas entrevistas fueron realizadas entre Diciembre de 2011 y Enero de 2012. La información cualitativa obtenida permitió dilucidar sobre la principal hipótesis de trabajo de este artículo, la cual es tomada de la noticia citada al inicio de esta sección y que señala - aunque de manera implícita - la existencia de una incipiente etnogentrificación en el sector oriental del Distrito de Collblanc - La Torrassa de L'Hospitalet de Llobregat. En lo que se refiere a las fuentes de información demográfica, los datos estadísticos primarios referidos a los stocks de población extranjera de derecho en el área de estudio definida para este artículo se encuentran desagregados por secciones censales y han sido facilitados tanto por el Departamento de Estadística del Ayuntamiento de L'Hospitalet de Llobregat como por el Instituto de Estadística de Catalunya. Los años seleccionados son 1999, 2004 y 2009, ${ }^{\text {vii }}$ de cinco en cinco, para facilitar el tratamiento estadístico de los datos.

\section{MARCO GEOGRÁFICO Y SOCIODEMOGRÁFICO DEL DISTRITO DE COLLBLANC - LA TORRASSA}

Situado en la mitad meridional de la primera corona metropolitana de Barcelona, L'Hospitalet constituye actualmente la segunda ciudad de Catalunya en número de habitantes (266.973) y la primera en densidad poblacional (22.249 hab./ $\left./ \mathrm{km}^{2}\right)$, según datos del Padrón municipal así como del Instituto de Estadística de Catalunya. Desde inicios del siglo XX, tanto la industrialización de los centros urbanos españoles como un conjunto de factores diversos que perjudicaron la estructura productiva 
del campo ibérico viii ya desde las dos últimas décadas del siglo XIX, favorecieron el primer flujo migratorio interno importante en España del campo a la ciudad. En este contexto cabe considerar L'Hospitalet de Llobregat como ciudad de acogida de los primeros inmigrantes que - a lo largo de las primeras décadas del siglo XX y venidos por primera vez desde fuera de Catalunya (principalmente desde Murcia y Almería, regiones del sureste español) - constituyeron una parte muy significativa de la mano de obra dedicada a la construcción de la primera línea de metro de Barcelona en 1924 por parte de la empresa Gran Metropolitano de Barcelona, S.A. Buena parte de este contingente inmigrante se alojó en barrios de autoconstrucción localizados en las pequeñas colinas cuaternarias tanto del llano de Barcelona como del margen izquierdo de la planicie deltaica del río Llobregat. Éste es el caso del barrio de La Torrassa del municipio de L'Hospitalet de Llobregat.
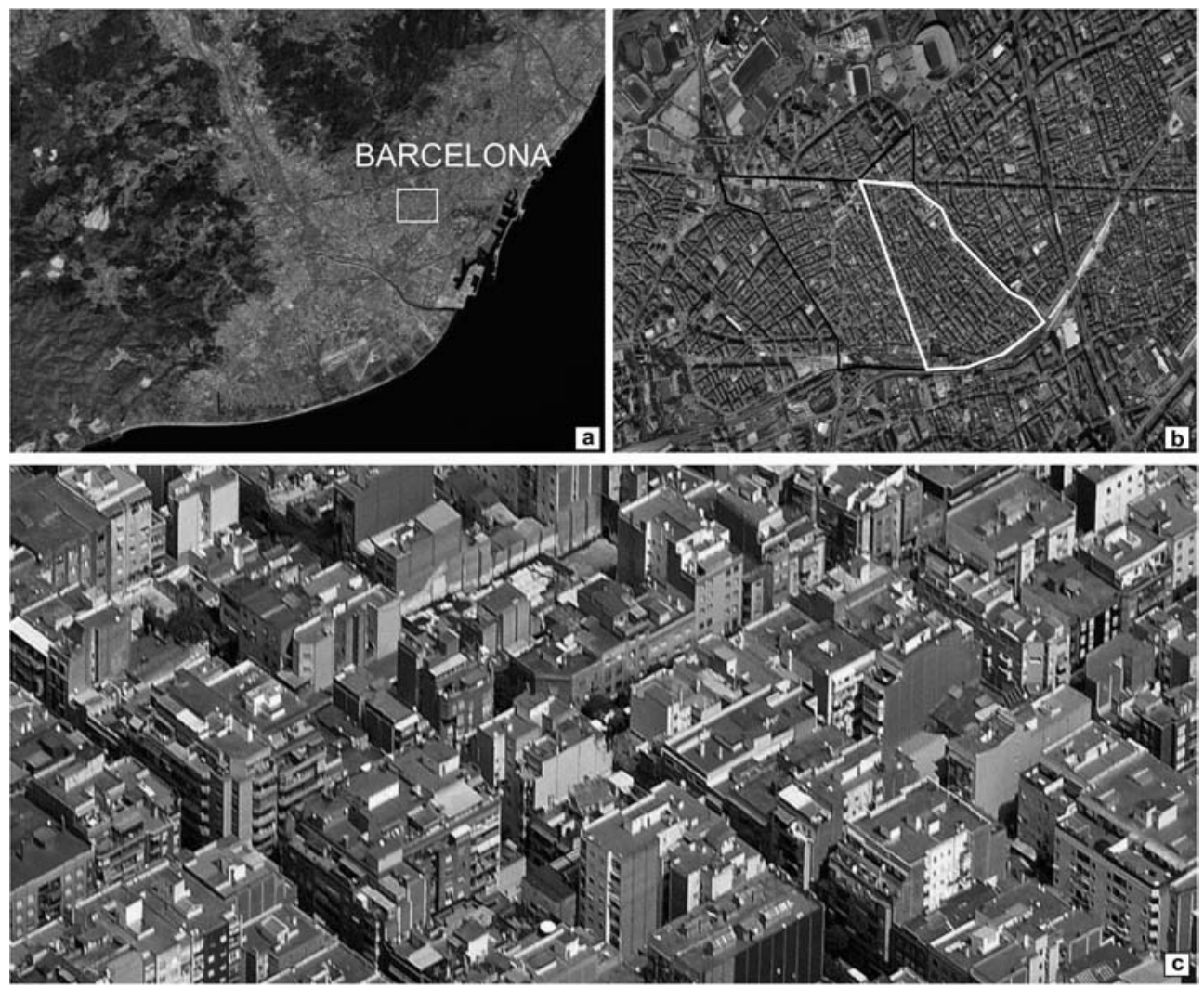

Fig. 1 - Mapa de localización (a) y detalle de la morfología urbana del barrio de La Torrassa de L'Hospitalet de Llobregat (c) Delimitación del límite administrativo del Distrito de Collblanc - La

Torrassa (en negro) y del área de estudio, el sector oriental de dicho distrito (en blanco) (b).

Fig. 1 - Location map (a) and detail of the urban morphology of the District of La Torrassa de L'Hospitalet de Llobregat (c) Delimitation of the administrative boundary of the District of Collblanc

- La Torrassa (in black) and of the study area, the eastern sector of the district (in white) (b). 
El origen auto-constructivo de las primeras edificaciones y el carácter extremadamente fragmentado de la propiedad del suelo agrario de esta área geográfica se erigen como una de las razones explicativas más importantes de las características edificatorias del barrio: la superficie media de la parcela residencial es de $184 \mathrm{~m}^{2}$ las líneas de fachadas son estrechas (el 39\%, con menos de 6,50 metros); el 32\% de las calles presentan una anchura de menos de 8 metros y el 31,50\% de las parcelas presenta edificaciones con alturas superiores a PB+4 (García, 2001). De hecho, más de la mitad de la edificación existente presenta unas características físicas alejadas de los parámetros legales establecidos por el planeamiento urbano local y metropolitano vigente (Íb.).

Después de la primera oleada inmigratoria del primer tercio del siglo XX, la penuria económica derivada de la Guerra Civil Española (1936-1939) comportó un nuevo flujo migratorio interno de notables proporciones, despoblando aún más la llamada España rural. Esta nueva oleada inmigratoria (1950-1975) consolidó a L'Hospitalet de Llobregat como la segunda ciudad de Catalunya en número de habitantes. El fenómeno migratorio y la condición de la misma L'Hospitalet como una de las ciudades de acogida y arraigo de los inmigrantes recién llegados a Catalunya comportaron la consecución de una densidad poblacional superior incluso a la capital catalana, Barcelona.

Durante los últimos diez años y a raíz de la última oleada inmigratoria en Catalunya, de claro carácter extracomunitario, L'Hospitalet de Llobregat se ha caracterizado por ser el municipio que, aparte de Barcelona, ha acogido más inmigrantes extracomunitarios de Catalunya. Dejando a un lado la capitalidad de la ciudad condal y su consecuente papel de puerta de entrada de la inmigración no europea en Catalunya, L'Hospitalet de Llobregat cuenta actualmente con 73214 habitantes nacidos fuera de España, un 27,42\% de su población de derecho, según datos del Padrón Municipal a fecha de 31 de Diciembre de 2011. Sin embargo, y como ya es de suponer, esta distribución no es homogénea en el territorio. Así, al 14,47 \% de población extranjera de su centro histórico (tradicionalmente habitado por medias y medias-altas autóctonas ya desde finales del siglo XVIII), habría que contraponerle porcentajes como el del barrio de La Florida (38,49\%), el del barrio de Collblanc $(35,52 \%)$ o el $41,10 \%$ del barrio de La Torrassa con una densidad poblacional de $61675 \mathrm{hab} / \mathrm{km}^{2}$.

El fuerte incremento entre los años 1996 y 2005 de nuevas altas de población extranjera en el padrón municipal de L'Hospitalet de Llobregat (44 4431, según datos del padrón de 2005) además de su condición ya mencionada como la segunda ciudad de acogida de inmigrantes en Catalunya y la existencia de un Estado del Bienestar débil y en claro proceso de retroceso (Navarro, 2006) ha comportado la eclosión de ciertas problemáticas urbanas hasta entonces - si bien no desconocidas - no muy extendidas en las ciudades catalanas y sus suburbios. En este sentido, la expansión durante los últimos diez años de la llamada infravivienda a través principalmente de la conversión de antiguos comercios, pequeños almacenes o talleres 
manufactureros y la posterior adquisición de la cédula de habitabilidad facilitada por la Dirección General de Vivienda del Gobierno de Catalunya (Nofre, 2006) se tornó una problemática urbana en algunos barrios del área metropolitana de Barcelona puesto que densificaba algunos de sus barrios ya entonces altamente congestionados, caracterizados por una crónica falta de equipamientos y servicios públicos y con frecuentes problemas de convivencia entre inmigrantes y autóctonos (ambos colectivos pertenecientes a las capas bajas de la sociedad barcelonesa metropolitana).

\section{CONTEXTUALIZANDO EL COMERCIO MINORISTA EN EL DISTRITO DE COLLBLANC - LA TORRASSA}

La rigidez de la oferta del comercio minorista autóctono en el Distrito de Colblanc - La Torrassa, especialmente de su sector alimentario - traducida esta rigidez en su escasa oferta de productos latinos, africanos o asiáticos - junto con la sobrevaloración del precio de los traspasos de locales comerciales y una cierta desconfianza mutua entre inmigrantes y autóctonos, facilitó la eclosión de un circuito comercial minorista étnico el cual se basaba en ofrecer productos propios del país o la región de origen del nuevo comerciante. Ello conllevó a que estos nuevos comercios minoristas del sector alimentario se convirtieran en puntos de apoyo para la creación de redes de cooperación y solidaridad entre colectivos inmigrantes con origen geográfico similar, a la vez que segmentaba el consumo según lugar de nacimiento: la clientela autóctona prefería más que nunca consumir en la tienda de siempre mientras que, para los inmigrantes sudamericanos (por ejemplo), esa nueva tienda de productos latinos constituía su punto de encuentro y contacto social en el barrio, elemento fundamental en la construcción de la familia extensa del individuo (Nofre, 2006).

Todo ello ha conllevado a una progresiva segmentación espacial del consumo cotidiano, su etnificación, constituyendo hoy día una de las características fundamentales del paisaje comercial minorista en el Distrito de Collblanc - La Torrassa de L'Hospitalet de Llobregat (Nofre, 2006). A ello cabe sumarle la aparición de elementos proto-gentrificadores, como locales de restauración y ocio nocturno orientados capas medias-altas metropolitanas, en el sector oriental del distrito de Collblanc - La Torrassa de L'Hospitalet de Llobregat constatado a partir del trabajo de campo realizado en 2004 (Nofre, 2006) y que respondería a la agenda oculta de renovación social recogida por el Plan de Rehabilitación Integral de Collblanc - La Torrassa (Nofre, 2009).

Sin embargo, la construcción de un barrio multicultural y atractivo para nuevos residentes más jóvenes con mayor poder adquisitivo no ha acabado de cuajar. Sea por la aparición de nuevas estrategias residenciales de los jóvenes de Barcelona y su Área Metropolitana (Costas, 2007), sea por la atracción de nuevas centralidades 
urbanas metropolitanas con excelente conectividad y accesibilidad a nivel local y regional, sea por la crisis económica y financiera o sea por la falta de expectativas y la incertidumbre del mañana asociada a la ecología del miedo que caracteriza las sociedades occidentales contemporáneas (Davis, 1992), o incluso sea por la imprevisibilidad de los flujos migratorios internacionales y su impacto en territorios urbanos, la substitución de la clase trabajadora autóctona del Distrito de Collblanc - La Torrassa por miembros con una posición social mayor y asociados a profesiones liberales no se ha producido en la dirección deseada por la administración pública local (Nofre, 2006). Nada más lejos que ello. Pero volvamos al objeto de estudio principal de este artículo - el comercio minorista étnico en el sector oriental del Distrito de Collblanc - La Torrassa - tomando como premisa la noticia citada en la introducción de este texto.

\section{DE GUAYAKIL A ISLAMABAD. ETNOGENTRIFICACIÓN O REORDENACIÓN DEL PAISAJE COMERCIAL MINORISTA?}

Como ya fue apuntado en el inicio de este artículo, la noticia aparecida el Martes 14 de Diciembre de 2010 en el rotativo barcelonés La Vanguardia acerca del cierre de negocios en el distrito de Collblanc - La Torrassa por parte de inmigrantes latinoamericanos y la consideración de L'Hospitalet como oportunidad de negocio para el colectivo pequeño-burgués pakistaní e hindú afincado en el centro de Barcelona permitiría elaborar una primera hipótesis de trabajo acerca de la existencia de una incipiente etnogentrificación en el barrio hospitalense. ¿Realmente es así?

Antes de iniciar la explicación detallada acerca de la posible etnogentrificación del sector oriental del Distrito de Collblanc - La Torrassa de L'Hospitalet de Llobregat, cabría destacar, aunque sea de manera somera, el nuevo contexto demográfico del área de estudio y los cambios acaecidos en diez años. Si bien en 1999, sólo un 2,87\% de la población del área de estudio había nacido fuera de España (584 individuos sobre un total de población de 20345 hab.), en 2009 el colectivo inmigrante constituía un $47,02 \%$, con 7815 individuos sobre un conjunto población de nuestra área de estudio de 16548 habitantes. Ante estos datos, cabría destacar aún más dos hechos: en primer lugar, la pérdida de 3797 habitantes de origen autóctono (españoles y catalanes); y en segundo lugar, el aumento en 7161 individuos del contingente inmigrante residente en el sector oriental del Distrito de Collblanc - La Torrassa. Es decir, mientras que el stock de inmigrantes residentes en la área de estudio definida para esta investigación aumentó un $226,20 \%$ en el período ya citado entre 1999 y 2009 , el volumen demográfico del colectivo autóctono registró una variación negativa, del $-18,66 \%$ en el mismo período. 
Tabla I - Principales colectivos de inmigrantes según su lugar de nacimiento en el sector oriental del Distrito de Collblanc - La Torrassa de L'Hospitalet de Llobregat e variación entre 2004 e 2009.

Table I-Main groups of immigrants according to their place of birth in the eastern sector of the District of Collblanc - La Torrassa de L'Hospitalet de Llobregat and variation between 2004 and 2009.

\begin{tabular}{ccccc}
\hline & Sector Oriental DCT & $\begin{array}{c}\text { Sector Oriental DCT } \\
\text { Variación (abs). }\end{array}$ & $\begin{array}{c}\text { \%Variación } \\
2004-09\end{array}$ \\
\hline Ecuador & 2004 & 2009 & $504-09$ & 3,62 \\
Perú & 1.380 & 1.430 & 177 & 33,33 \\
Colombia & 531 & 708 & -32 & $-7,94$ \\
Pakistán & 403 & 371 & 200 & 157,48 \\
India & 127 & 327 & 172 & 160,75 \\
China & 107 & 279 & 115 & 135,29 \\
España & 85 & 200 & -1801 & $-9,82$ \\
\hline
\end{tabular}

\begin{tabular}{ccccc}
\hline & $\begin{array}{c}\text { L'Hospitalet } \\
2004\end{array}$ & $\begin{array}{c}\text { L'Hospitalet } \\
2009\end{array}$ & $\begin{array}{c}\text { Variación (abs). } \\
2004-09\end{array}$ & $\begin{array}{c}\text { \%Variación } \\
\text { 2004-09 }\end{array}$ \\
\hline Ecuador & 13400 & 13517 & 117 & 0,87 \\
Perú & 4103 & 5502 & 1399 & 34,10 \\
Colombia & 2974 & 3152 & 178 & 5,99 \\
Pakistán & 1439 & 3316 & 1877 & 130,44 \\
India & 899 & 2201 & 1302 & 144,83 \\
China & 1117 & 1978 & 861 & 77,08 \\
España & 212291 & 193785 & -18506 & $-8,72$ \\
\hline
\end{tabular}

Fuente: Datos censales (2011) facilitados por el Instituto de Estadística de Catalunya.

Sin duda alguna que esta nueva estructura demográfica del sector oriental del Distrito de Collblanc - La Torrassa entre 2004 e 2009 (tabla I) ha conllevado cambios substanciales en el paisaje urbano, entre ellos, el del comercio minorista y más concretamente, el del comercio étnico. Además, hay cabría señalar que mientras el contingente ecuatoriano aumenta ligeramente $(3,62 \%)$, el peruano moderadamente $(33,33 \%)$ e incluso el colombiano registra una variación poblacional negativa $(-7,94 \%)$, los pakistaníes $(157,48 \%)$, los hindúes $(160,75 \%)$ y los chinos $(135,29 \%)$ registran unas variaciones positivas extraordinarias .

No cabe duda que tales cambios demográficos conducirían a plantearse, aunque de manera hipotética, cambios significativos en el paisaje comercial minorista étnico del área de estudio. Efectivamente, el trabajo de campo realizado los días 4,5 y 7 de Enero de 2011 permitió constatarlo meridianamente. Como ya fue apuntado en la introducción de este texto, esta segunda oleada del trabajo de campo observacional permite afirmar que el número de locales de comercio étnico regentados por pakistaníes e hindúes han aumentado un 137,04\%, pasando de un total de 21 locales en Diciembre de 2004 a 64 en Enero de 2011. En lo que se refiere a su distribución espacial, el 50,44\% de los nuevos locales de comercio étnico regentados por pakistaníes e/o hindúes se localizan en tan sólo tres calles: la calle Llobregat, la calle Martí Julià y la calle Holanda (tabla II, fig. 2). 


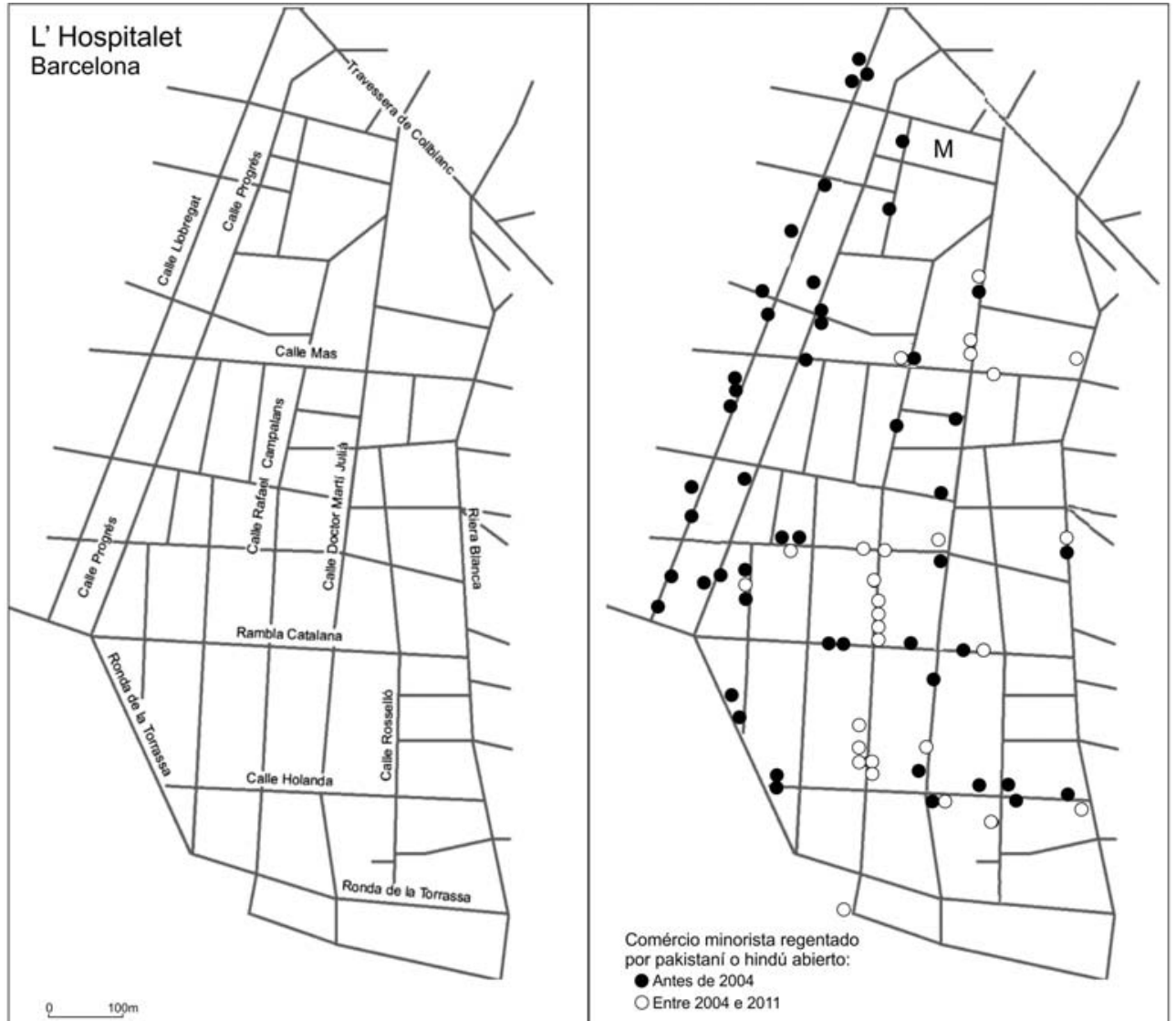

Fig. 2 - Comercio pakistaníes y/o hindúes en la área de estudio en 2004 e 2011.

Fig. 2 - Retail stores run by Pakistan and/or Hindu citizens in the study area in 2004 and 2011.

Fuente: Elaboración a partir de trabajo de campo (2011)

Tabla II - Comparación de la información de la figura 2.

Table II - Comparison of the data of figure 2.

\begin{tabular}{rccc}
\hline Typology & 2004 & 2011 & $\begin{array}{c}\% \text { var. } \\
2004 / 11\end{array}$ \\
\hline Call Centres & 10 & 21 & 110 \\
Hairdressers & 3 & 4 & 33,33 \\
Others & 0 & 7 & - \\
\hline Total & 27 & 64 & 137,04 \\
\hline
\end{tabular}

En lo que se refiere a la tipología de este nuevo comercio, hoy en día casi el $75 \%$ de los negocios étnicos del sector oriental del distrito de Collblanc - La Torrassa regentados por pakistaníes e hindúes pertenecen tanto al ramo comercial de la alimentación y bebidas como al de los servicios telefónicos y de Internet. Los pe- 
queños supermercados y los colmados (genéricamente agrupados en la categoría "Alimentación y Bebidas" para facilitar la homogeneización de los datos en su trato geoestadístico) presentan el aumento más sustancial, con un 225\% (de 8 a 26 locales), mientras que en segundo lugar se encuentra el aumento del número de locutorios, con un $110 \%$ respecto a Diciembre de 2004 (de 10 a 21). Pero ello no quiere decir que el paisaje minorista del barrio tienda a una cierta homogeneización como sugiere el titular apuntado por el rotativo barcelonés La Vanguardia.

Una lectura detallada del mapa del comercio étnico en el sector oriental del Distrito de Collblanc - La Torrassa de L'Hospitalet de Llobregat en 2004 (Nofre, 2006) junto con la evolución espacio-temporal del comercio minorista regentados por pakistaníes y/o hindúes en entre 2004 y 2011 (fig. 2) permite ver más allá de lo simplemente afirmado por La Vanguardia acerca de la compra de locales comerciales por parte de pakistaníes e hindúes a inmigrantes latinoamericanos que optan por volver a su país de origen. De hecho, el crecimiento espacial registrado entre 2004 y 2011 del comercio minorista regentado por el colectivo pakistaní e hindú ha tendido en concentrarse en aquel subsector de nuestra área de estudio en donde el comercio minorista latinoamericano tenía una menor presencia en 2004 - es decir, a lo largo de las calles Llobregat y Progrés las cuales vertebran la movilidad interna cotidiana tanto peatonal como motorizada en el sector occidental de nuestra área de estudio. En cambio, el comercio minorista étnico latinoamericano se concentraba, en 2004, en la mitad meridional del sector oriental del distrito de Collblanc - La Torrassa (es decir, en aquella área comprendida entre las calles Rambla Catalana y Ronda de la Torrassa). Ello permitiría afirmar que si bien no cabe menospreciar algún tipo de tendencia en el mercado de compra y/o alquiler de locales comerciales de pequeño tamaño entre ambos colectivos, no sería arriesgado afirmar que el colectivo pakistaní e hindú ha priorizado fomentar la complementariedad de la oferta minorista étnica frente a una política comercial minorista agresiva que se hubiera aprovechado de los primeros indicios de retorno del colectivo latinoamericano a su país de origen.

A todo ello cabría añadir otras informaciones que apriorísticamente no guardarían una relación directa con la distribución espacial de los nuevos comercios étnicos de pakistaníes e hindúes en La Torrassa: a) el abandono de actividades comerciales y pequeño-manufactureras por parte del colectivo autóctono, poniendo a la venta o en alquiler aquellos locales donde desarrollaban su actividad; b) el "muro" invisible que constituye el clúster de comercio minorista autóctono vertebrado alrededor del Mercado Municipal de Collblanc, tratándose de un sector impermeable a foráneos y que aún sigue controlado por familias relacionadas con su inauguración en 1934; y c) la concentración de población autóctona envejecida de más de 65 años en la mitad meridional del área de estudio en cuestión.

Si bien los nuevos locales de comercio étnico de pakistaníes e hindúes han conseguido penetrar en el eje de comercio minorista autóctono de la calle Martí i Julià, su expansión espacial se ha realizado sobre todo a lo largo de la calle Llobregat como 
ya ha sido anteriormente mencionado. Este hecho no debería sorprender atendiendo a la evolución de precios del mercado inmobiliario en el barrio durante los cinco últimos años. A partir de la información facilitada por la agencia inmobiliaria que la empresa Don Piso, S.A. tiene ubicada en la misma calle Martí i Julià frente al Mercado Municipal de Collblanc y tomando como ejemplos los pisos en venta de segunda mano a fecha primera semana de Enero de 2011, el precio del $\mathrm{m}^{2}$ construido bajo techo es un 21,51\% menor que el precio medio de La Torrassa $\left(3057,76 € / \mathrm{m}^{2}\right)^{\mathrm{ix}}$. La expansión de los negocios pakistaníes e hindúes hacia el margen occidental del barrio conjuntamente con el paulatino cierre de negocios y comercios regentados por sudamericanos y el abandono masivo de actividades comerciales y manufactureras de los autóctonos - ya citado anteriormente - ha conllevado un estado residual de la actividad económica no inmobiliaria tanto en el extremo suroriental Distrito (vertebrado por la calle Ronda de La Torrassa).

Por otra parte cabría citar las concentraciones elevadas de población mayor de 65 años en la mitad meridional del sector estudiado como posible factor explicativo de la particular vertebración espacial del comercio étnico pakistaní e hindú: ello se debería a la desconfianza de los primeros hacia los comerciantes "no autóctonos", y a la "rápida percepción" de los comerciantes pakistaníes e hindús que, efectivamente, este segmento de la población no constituye su principal target. Según Ángeles García (2001) en su trabajo analítico sobre la renovación integral del Distrito de Collblanc - La Torrassa, el mayor porcentaje de personas del grupo etario citado se daban en el extremo meridional del área de estudio considerada en este texto (donde precisamente se concentran la mayor parte de los locales en alquiler, como ya ha sido comentado) con concentraciones que varían entre el 30 y el $45 \%$ así como en el tramo final de la Calle Rosselló, área en que el porcentaje asciende a valores comprendidos entre el $45 \%$ y el $70 \%$.

No debería ser pasado por alto el hecho que, tal y como ha sido comentado en la primera mitad del artículo, cada entorno social promueve su propia clase de racionalidad y orden, reforzando el vínculo entre pureza y seguridad de la comunidad como estrategia fundamental en la generación de redes sociales dentro de cada colectivo: los consumidores autóctonos de más de 65 años no suelen comprar en los negocios y comercios étnicos. De ahí también la etnificación del espacio público y del consumo cotidiano. Y de ahí también la expansión del comercio pakistaní e hindú en aquellos subsectores del área de estudio donde residen personas más jóvenes $\mathrm{y}$, sobretodo, inmigrantes extracomunitarios que se convierten, indudablemente, en potenciales consumidores de este tipo de negocios y comercios étnicos, mucho más que sus conciudadanos autóctonos. El abandono de las actividades económicas tradicionales por parte de pequeños propietarios autóctonos podría ser englobado en el contexto de "huida" protagonizado por este colectivo. Así, en el período 1986-2009, una parte significativa del colectivo autóctono (los primeros que llegaron al barrio a partir del primer tercio de del siglo XX) han emigrado del barrio: concretamente, un 18,66\% entre 1999 y 2009 (3 797 individuos de los 20345 empadronados en 1999). 
Sin embargo, el hecho que los pakistaníes e hindúes comiencen a adquirir estos locales vacíos ${ }^{x}$ no debería ser considerado en un contexto etnogentrificador como sugería implícitamente la noticia del rotativo barcelonés La Vanguardia y como la aplicación de los estudios realizados para el caso del barrio del Raval de la capital catalana sugeriría hipotéticamente para el sector oriental del Distrito de Collblanc La Torrassa. Si bien esta desinversión y "huida" por parte de los colectivos autóctono y suramericano y la creciente inversión en pequeños negocios y comercios por parte de diferentes colectivos asiáticos podría ser considerada como condición necesaria para la (etno)gentrificación del barrio según las diferentes tesis economicistas y culturalistas presentadas en la introducción de este texto, las diferencias entre los casos tratados principalmente por la academia anglosajona y el caso estudiado en este texto sugerirían una re-definición del fenómeno: no se trataría tanto de un proceso etnogentrificador como sí de una reordenación del paisaje comercial minorista étnico derivada de una sustitución poblacional lenta pero progresiva.

\section{CONCLUSION}

A menudo Barcelona es conocida mundialmente por sus atractivos turísticos. A la vez que es conocida por su arquitectura y su oferta de ocio y cultura, la capital de Catalunya aparece como una ciudad semi-periférica global en diversos rankings urbanos internacionales (Taylor, 2004). Como tal, presenta problemas comunes a las ciudades posfordistas occidentales y también problemas sociales, económicos, culturales e incluso políticos que mucho tienen que ver con inercias históricas particulares. El mundo científico-académico local - y últimamente también internacional - no es ajeno a estas problemáticas que revisten de sumo interés por lo que ha significado la exportación del llamado "Modelo Barcelona" en la última oleada de renovaciones urbanas de ciudades, por ejemplo, latinoamericanas. Sin embargo, gran parte de estos estudios tratan Barcelona de manera aislada, desconsiderando el hecho que actualmente la ciudad condal no puede ser entendida sin su suburbanización y su posterior metropolitanización.

El abanico de estudios acerca de la(s) problemática(s) asociadas al comercio étnico de la capital catalana ejemplifican la denuncia realizada en este texto. Muchos son los trabajos publicados acerca del Raval y su comercio étnico: muchas conferencias, muchos seminarios han sido llevados a cabo, los cuales (indefectiblemente) tienen la función oculta de reproducir esta visión "modélica" de Barcelona y las soluciones aportadas desde el inmenso laboratorio urbano de generación y exportación de nuevas "soluciones urbanas" en la que se han convertido los diferentes espacios de reflexión y discusión, como la propia universidad. Sin embargo, la producción bibliográfica acerca de las problemáticas de barrios conflictivos como Vallbona, Torre Baró, Bon Pastor (todos ellos de Barcelona), o Llefiá (de Badalona), Sant Cosme (El Prat de Llobregat) o la misma La Torrassa (L'Hospitalet) es escasa y de calidad desigual, si no prácticamente nulas. 
Este artículo ha presentado una cartografía comparativa entre los años 2004 y 2011 de la evolución del comercio minorista étnico en el sector oriental del Distrito de Collblanc - La Torrassa de L'Hospitalet de Llobregat (ciudad suburbana del Área Metropolitana de Barcelona), focalizando en la adquisición de nuevos negocios comerciales étnicos regentados por pakistaníes e hindúes que han sido comprados recientemente a antiguos propietarios sudamericanos, quienes están volviendo a su país de origen ante la aguda crisis económica española y la mejora de la situación socioeconómica de sus países de origen.

El caso de estudio presentado en este artículo reviste de una gran singularidad, la cual radica en poner en cuestión la aplicación en contextos urbanos mediterráneos - como es el caso que ha ocupado este texto - de las diferentes tesis economicistas y culturalistas sobre etnogentrificación elaboradas principalmente desde la academia anglosajona (Shaw et al., 2004; Airriess, 2006; Smith, 2006; o Godfrey, 2007; entre otros). En este sentido, este artículo ha pretendido advertir al lector que el uso del término "etnogentrificación" strictu sensu en el caso del estudio del sector oriental del distrito de Collblanc - La Torrassa de L'Hospitalet de Llobregat podría no ajustarse del todo a los resultados ofrecidos gracias al trabajo de campo realizado y a su cartografía derivada. Y es que la aplicación de un concepto surgido del ámbito científico anglosajón en un contexto geográfico diferente, sin mediar matiz alguno, puede conllevar la generación de ciertas inexactitudes léxicas. Es por ello que, como se ha sugerido en la segunda parte del texto, lo estudiado a lo largo de este artículo no constituiría tanto un proceso etnogentrificador como sí de una reordenación del paisaje comercial minorista étnico derivada de una sustitución poblacional lenta pero progresiva.

Como última nota cabría mencionar que, a pesar que no existe información primaria certeramente validada sobre la posición social, la renta familiar bruta disponible y el capital educativo de los inmigrantes y sus familias, las entrevistas informales off the record llevadas a cabo durante los días en que se realizó el trabajo de campo permitieron constatar ciertas ventajas del colectivo pakistaní e hindú sobre los latinoamericanos: un mayor nivel instructivo y una mayor integración sociolingüística en la lengua propia de Catalunya, el catalán (elemento clave para la ascensión social ${ }^{\mathrm{xi}}$. Pero también permitieron certificar, desde un tercer actor, otro fenómeno ciertamente interesante: el fracaso de la integración social del colectivo sudamericano y el creciente conflicto de convivencia entre este colectivo y el autóctono. ${ }^{\text {xii }}$ Esta percepción por parte del colectivo pakistaní e hindú sobre el colectivo latinoamericano deja entrever claramente cierta fractura en la cohesión social del barrio. Sin duda alguna, la vinculación entre esta reordenación del paisaje comercial minorista étnico del sector oriental del Distrito de Collblanc - La Torrassa de L'Hospitalet de Llobregat y el reforzamiento de los mecanismos de pureza y seguridad de la comunidad se antoja como un campo de estudio a seguir explorando ciertamente interesante y de notable alcance en la exploración de la reciente fractura social que caracterizan los espacios suburbanos de Barcelona densamente congestionados por población inmigrante de perfil socioeconómico bajo. 


\section{AGRADECIMIENTOS}

Esta investigación ha sido llevada a cabo gracias a la presente financiación del Gobierno de Portugal (Fundação para a Ciência e a Tecnologia) y a la pasada financiación del Gobierno de Catalunya (Agència de Gestiód'AjutsUniversitaris i Recerca, período 2005-2008). Agradezco el apoyo logístico del Centro de Estudos de Sociologia da Universidade Nova de Lisboa (CesNova-FCSH/ UNL). También quisiera agradecer a Pat Gràcia y a Dolors Olivares, del Instituto de Estadística de Catalunya y a Marina Valor del Departamento de Estadística del Ayuntamiento de L'Hospitalet por la desagregación territorial de los datos estadísticos relativos al área de estudio. Finalmente, también quisiera agradecer de antemano los comentarios, críticas, correcciones y mejoras señaladas por los revisores de este artículo.

\section{BIBLIOGRAFÍA}

Airriess C A (2006) Scaling central place of an ethnic-vietnamese commercial enclave in New Orleans, Louisiana. In Kaplan D, Wei L (eds.) Landscapes of the ethnic economy. Rowman \& Littlefield, Plymouth (UK): 17-33.

Barret G, McEvoy D (2006) The evolution of Manchester's curry mile: from suburban shopping street to ethnic destination. In Kaplan D, Wei L (eds.) Landscapes of the ethnic economy. Rowman \& Littlefield, Plymouth (UK): 193-208.

Barth F (1969) Ethnic groups and boundaries. The social organization of culture difference. Little \& Brown, Boston.

Beltrán Antolín J (2000) Expansión geográfica y diversificación económica. Pautas y estrategias del asentamiento chino en España. Actas del II Congreso sobre la Inmigración en España. España y las migraciones internacionales en el cambio de siglo. Instituto Universitario de Estudios sobre Migraciones de la Universidad Pontificia Comillas/Instituto Universitario Ortega y Gasset, Madrid.

Beltrán Antolín J, Saíz López A (2007) Nouvelles circulations, activités économiques et présence asiatique dans les villes espagnoles. In Berry Chikhaoui I, Deboulet A, Roulleau-Berger L (eds) Villes internationales. Entre tensions et réactions des habitants. La Découverte, París: 263-277.

Bonacich E (1973) A theory of middlemen minorities. American Sociological Review, 38: 585-594.

Bryman A (2004) The disneyzation of society. Routledge, London.

Costas A (ed) (2007) El llibreblanc de l'habitatge. Consell de la Ciutat, Barcelona.
Davis M (1992) Beyond blade runner: urban control, the ecology of fear. Westfield, New Jersey.

Ferrer Regalés M (2006) Distribución, difusión y centralidad musulmanas en España. Estudios Geográficos, 261: 495-521.

García A (2001) Indicadors per a un Pla Integral a Collblanc i La Torrassa. Papers de la Regió Metropolitana de Barcelona, 33. Disponible en: http://www.raco.cat/index.php/PapersIERMB/ article/view/103882/130044 [Accedido en: 22 de Diciembre de 2010].

Harvey D (2005) A brief history of neoliberalism. Oxford UP, New York.

Kaplan D (1998) The spatial structure of urban ethnic economies. Urban Geography, 19(6): 489-501.

Kesteloot C, Mistiaen P (1997) From ethnic minority niche to assimilation: Turkish restaurants in Brussels. Area, 29(4): 325-334.

Kloosterman R, van Der Leun J, Rath J (1999) Mixed embeddedness: (in)formal economic activities and immigrant business in the Netherlands. International Journal of Urban and Regional Research, 23(2): 253-267.

Krase J, Hum T (2006) Immigrant global neighborhoods: perspectives from Italy and the United States. In Hutchison R, Krase J (eds) Ethnic landscapes in an urban world. Elsevier, Atlanta: 97-120.

Light I, Gold S (2000) Ethnic economies. Academic Press, San Diego.

MaMung E, Guillon M (1986) Les commerçants étrangers dans l'agglomération parisienne. Revue Européenne des Migrations Internationales, 2(3): 105-134. 
Li W, Dymski G, Chee M, Ahn H, Aldana C, Zhou Y (2006) How ethnic banks matter: banking and community/economic development in Los Angeles. In Kaplan D, Wei L (eds.) Landscapes of the Ethnic Economy. Rowman\& Littlefield, Plymouth (UK): 113-134.

Loda M, Mancini N (2003) Imprenditoriaextracomunitaria e commercio al dettaglioneicentristorici. Ilquartieredi San Lorenzo a Firenze. In Loda $\mathrm{M}$, Clemente $\mathrm{P}$ (eds.) Migrare a Firenze. Comunedi Firenze \& Università degli Studi, Firenze: 123-148.

López Sala A M, Esteban Sánchez V (2010) Los comerciantes sindhis en Canarias: relaciones transnacionales y actividad económica. Revista CIDOB d'afers internacionals, 92: 139-159.

Martínez Rigol S (2001) El retorn al centre de la ciutat. La reestructuracio del Raval, entre la renovacio $i$ la gentrificacio. Tesis doctoral. Departamento de Geografía Humana, Universidad de Barcelona.

Miranda A (2007) Naples. Ethnicisation et circulations migratoires dans un contexte urbain méditerranéen. In Berry Chikhaoui I, Deboulet A, Roulleau-Berger L (eds.) Villes Internationales. Entre tensions et réactions des habitants. La Découverte, Paris: 279-297.

Navarro V (2006) El subdesarrollo social de España. Causas y consecuencias. Barcelona: Anagrama.

Nofre J (2011) Youth policies, social sanitation and contested suburban nightscapes. Research in Urban Sociology, 11: 261-281.

Nofre J (2009) L'Agenda Cultural Oculta. Una deconstrucció de l'oci nocturn de Barcelona $i$ els seus suburbis. Premi Joventut 2009. Tesis Doctoral. Universitat de Barcelona.

Nofre J (2006) El problema de la vivienda y la inmigración en la periferia de Barcelona. El caso de La Torrassa. In Carreras C, Fani A (eds). Barcelona - Sao Paulo, cara a cara. Procesos metropolitanos a la hora de la globalización. Editorial Da Vinci, Barcelona: 185-196.

Raulin A (2000) L'ethnique est quotidien: diasporas, marchés et cultures métropolitaines. Connaissance des hommes, Paris.
Rekers A, van Kempen R (2000) Location matters: ethnic entrepreneurs and the spatial context. In Rath J (ed) Immigrant business: the economic, political, and social environment. Palgrave MacMillan, New York: 54-69.

Riesco Sanz A (2007) Dimensiones y características de la empresarialidad inmigrante en Lavapiés (Madrid). In Actas del IV Congreso de la Inmigración. Universidad de Valencia, Valencia.

Serra P (2006) El comercio étnico en el distrito de Ciutat Vella de Barcelona. Barcelona: Fundació La Caixa.

Shaw S, Bagwell S, Karmowska J (2004) Ethnoscapes as spectacle: reimaging multicultural districts as new destinations for leisure and tourism consumption. Urban Studies, 41(10): 1983-2000.

Smith J (2006) Little Tokyo. Historical and contemporary Japanese American identities. In Frazier J, Tettey-Fio E (eds.) Race, Ethnicity, and Place in a Changing America. Global Academic Publishing, Binghamton. 301-308.

Taylor I (2000) European ethnoscapes and urban redevelopment. The return of Little Italy in $21^{\text {st }}$ century Manchester City, 4(1): 27-42.

Taylor PJ (2004) World city network: a global urban analysis. Routledge, London.

Torres Pérez F (2007) Nous veïns a la ciutat. Els immigrants a València i Russafa. Tesis doctoral. Universitat de València, Valencia (España).

Valenzuela H (2010) Pecunia Ex Machina, el emprendedor pakistaní en la ciudad de Barcelona. Revista CIDOB d'afers internacionals, 92: 185-206.

Waldinger R (1999) Not the promised city: Los Angeles and its immigrants. Pacific Historical Review, 68(2): 253-272.

Waldinger R (1993) The ethnic enclave debate revisited. International Journal of Urban and Regional Research, 17(3): 444-452.

Wilson KL, Portes A (1980) Immigrant enclaves: an analysis of the labor market experiences of Cubans in Miami. American Journal of Sociology, 86: 295-319.

Zhou M (1992) New York's Chinatown: the socioeconomic potential of an urban enclave. Temple University Press, Philadelphia.

i Guayakil es la principal ciudad de Equador, aunque no capital

ii Raval es el nombre del barrio que ocupa la mayor parte de la mitad occidental del centro histórico de Barcelona.

iii Información facilitada por A.B., director de oficina bancaria agenciada en el barrio de La Torrassa de L'Hospitalet de Llobregat. Entrevista realizada el 20 de diciembre de 2010 en la misma oficina. 
iv El debate suscitado acerca del "modo" de integración de los diferentes colectivos inmigrantes en las grandes ciudades norteamericanas como Los Ángeles es de un gran interés e intensidad. Como ejemplo de ello cabría destacar la respuesta que Roger Waldinger $(1993,1999)$ ofrece al trabajo de A. Portes y K.L Wilson (1980) sobre las experiencias laborales de los cubanos en Miami, contraponiendo el concepto de "economía étnica" al de "enclave económico étnico". En su crítica, Waldinger sugería que aquellos que Portes y Wilson observaban en un "reducto" espacial de la ciudad, también se reproducía a lo largo y ancho de la urbe, dejando de este modo la variable espacial en una posición irrelevante en la explicación del fenómeno de la economía étnica. El área definida como "sector oriental del Distrito de Collblanc - La Torrassa" es aquella delimitada por las calles

v El área definida como "sector oriental del Distrito de Collblanc - La Torrassa" es aquella delimitada por las calles Riera Blanc, Travessera de Collblanc, Carrer Llobregat y Ronda de la Torrassa (fig. 1).

vi La petición del número de locales regentados por ciudadanos según su nacionalidad y su lugar de nacimiento fue denegada por el Área de Promoción Económica del Ayuntamiento de L'Hospitalet, mientras que desde el Departamento de Estadísitica del mismo Ayuntamiento optó por hacer caso omiso de la petición. Por otra parte, el Instituto de Estadística de Catalunya respondió que no podía ofrecer esos datos, remitiendo al investigador de este artículo a contactar con el respectivo Ayuntamiento.

vii Tanto para 1999, como para 2004 y 2009, los datos son referidos a 31 de Diciembre de cada año respectivo.

viii Juan Piqueras Haba (2005) señala como la filoxera fue una de las principales causas del primer despoblamiento del campo español. Si bien la plaga de la filoxera que afectó gravemente los viñedos tuvo lugar en Catalunya entre 1879 y 1892, su difusión lenta a partir de los focos de Málaga y la frontera del valle del Duero con Portugal comportó que no remitiera hasta 1930. Sin duda alguna, ello comportó el primer éxodo rural hacia zonas urbanas de la España contemporánea.

ix Datos de la inmobiliaria Don Piso, S.A. referidos a la primera semana del mes de Enero de 2011.

$x$ Información obtenida a partir de entrevista informal off the record con uno de los propietarios del Locutorio Aya, de Rambla Catalana 15, 9 de Enero de 2011, 16:10 p.m.

xi Esta es la tesis que ha mantenido siempre el ex-presidente del Gobierno Catalán Jordi Pujol y Soley, y la cual ha servido como línea ideológica principal de la política lingüística de Catalunya durante los últimos treinta años. Consúltese: http://www.jordipujol.cat/en/cejp/conferencies/173

xii Son constantes las quejas sobre faltas de convivencia asociadas a fiestas realizadas en horas nocturnas, volúmenes de música que sobrepasan las ordenanzas municipales, episodios de violencia juvenil y violencia doméstica y desórdenes públicos asociados a peleas y consumo excesivo de alcohol. Información facilitada off the record por agentes de la Policía Local de L'Hospitalet de Llobregat y del Cos de Mossos d'Esquadra de Catalunya. Entrevista realizada en la Unidad Móvil de Atención al Ciudadano de Rambla Just Oliveras, 17 de Enero de 2011, 11:32 a.m., registro manual. 